\title{
A moderate deviation for associated random variables
}

\author{
Tonguç Çağın ${ }^{\mathrm{a}, 1}$, Paulo Eduardo Oliveira ${ }^{\mathrm{a}, 1}$, Nuria Torrado $^{\mathrm{a}, 1,2}$ \\ ${ }^{a}$ CMUC, Department of Mathematics, University of Coimbra, 3001-454 Coimbra, Portugal
}

\begin{abstract}
Moderate deviations are an important topic in many theoretical or applied statistical areas. We prove two versions of a moderate deviation for associated and strictly stationary random variables with finite moments of order $q>2$. The first one uses an assumption depending on the rate of a Gaussian approximation, while the second one discusses more natural assumptions to obtain the approximation rate. The control of the dependence structure relies on the decay rate of the covariances, for which we assume a relatively mild polynomial decay rate. The proof combines a coupling argument together with a suitable use of Berry-Esséen bounds.
\end{abstract}

Keywords: moderate deviation, association, coupling, approximation 2010 MSC: 60E15, 60F10

\section{Introduction}

Sums of random variables have always been a central subject in the probabilistic literature, with a special interest on their asymptotics. Among results on this topic the important Central Limit Theorems (CLT) describes the limiting distributional behaviour of such sums, providing useful approximate descriptions of the tail probabilities. These, besides their natural theoretical interest, are extremely relevant in statistical applications. There is, however, a limitation inherent to the properties of convergence in distribution, requiring that the tails considered through the limiting process should be computed at

\footnotetext{
${ }^{1}$ This work was partially supported by the Centre for Mathematics of the University of Coimbra UID/MAT/00324/2013, funded by the Portuguese Government through FCT/MEC and co-funded by the European Regional Development Fund through the Partnership Agreement PT2020.

${ }^{2}$ This author is supported by the Portuguese Government through the Fundação para a Ciência e a Tecnologia (FCT) under the grant SFRH/BPD/91832/2012. 
points that behave like the variance. More specifically, if the random variables $X_{n}$, $n \geq 1$, are assumed centered and we define $S_{n}=X_{1}+\cdots+X_{n}, s_{n}^{2}=\mathrm{E} S_{n}^{2}$, the CLT provide the approximation of $\mathrm{P}\left(S_{n}>x s_{n}\right)$ by $N(x)=1-\Phi(x)$, for $x>0$ fixed, where $\Phi$ is the distribution function of a standard Gaussian variable. If we allow $x$ to depend on $n$, converging to infinity, then the approximation for $\mathrm{P}\left(S_{n}>x s_{n}\right)$ is known as a moderate or large deviation, depending on how fast $x$ grows to infinity, moderate deviations corresponding to the case where $x=O\left(s_{n}\right)$. In such cases where $x$ is growing to infinity, the approximating function $N$ is no longer necessarily the tail of a standard Gaussian, depending on the growth rate of $x$ to infinity.

First large deviations were proved by Linnik [11], Ibragimov and Linnik [10], Nagaev $[16,15]$ or Rozovski [27] for independent and identically distributed variables. We refer the reader to the survey paper by Nagaev [17] for a nice account of these early results. The techniques of proof were based on suitable exponential bounds, the so called Fuk-Nagaev inequalities, on the tail probabilities. A typical result, given in Theorem 1.9 in Nagaev [17], states that

$$
\mathrm{P}\left(S_{n}>x s_{n}\right)=\left(1-\Phi(x)+n \mathrm{P}\left(X_{1}>x s_{n}\right)\right)(1+o(1)),
$$

provided that $x \geq 1, s_{n}=n^{1 / 2}$ and the right tail of the $X_{n}$ 's is a regularly varying function. Extensions of such results have been recently proved by Peligrad, Sang, Zhong and $\mathrm{Wu}$ [25] considering weighted sums $\tilde{S}_{n}=\sum c_{n, i} X_{i}$ instead of $S_{n}$. These authors prove a result similar to (1) under essentially the same assumptions on the random variables (i.i.d. and regularly varying tails) and a regularity condition on the weights: $\max _{i} c_{n, i} / \mathrm{E} \tilde{S}_{n}^{2} \longrightarrow 0$. The proof of this extension relies on moderate or large deviations for triangular arrays of random variables and convenient strong approximations between the tails of $\tilde{S}_{n}$ and the sums of tails of the $X_{n}$ 's, much in the same spirit of the proof technique used in Theorem 1.9 in Nagaev [17]. Going back to early results, moderate or large deviations for triangular arrays of row-wise independent variables were considered by Rubin and Sethuraman [28], Amosova [1], Slastnikov [29] or, more recently, by Frolov [6]. All the results mentioned so far characterize the tail probabilities directly. Concerning large deviations, that is, $x$ growing fast to infinity, a lot of attention was given to the logarithms of the tail probabilities instead, thus providing exponential bounds for the tail probabilities themselves. The bound for these logarithms appears then as the 
Fenchel-Légendre transform of the normalized logarithm of the Laplace transform of $S_{n}$ (notice that we are now back to non-weighted sums). A good account of results in this direction can be found in the book by Dembo and Zeitouni [5]. The interest on logarithmic tails meant that there are much fewer results available in the non-logarithmic scale in recent literature, particularly for weighted sums. Another recent direction of development is concerned with dependent variables. Here, available results seem even more scarce. Looking at large deviations, some results were proved by Nummelin [21], Bryc [2] or Bryc and Dembo [3] considering mixing variables or, Henriques and Oliveira [9] for associated random variables. These authors were interest on logarithmic scale results and their proof techniques relied on suitable exponential bounds and required a rather fast decay rate on the coefficients characterizing the dependence structure, meaning they should decrease faster than geometrically. More recently, for mixing variables Merlevède, Peligrad and Rio [13] relaxed the assumption on the mixing coefficients, requiring only a geometric decay rate to prove the large deviation. Their proof technique, called by the authors a "Cantor set construction", adapts the block decomposition of sums, popular for proving CLT, to large deviations. These authors have more recently extended their results to other forms of weak dependent variables (see Merlevède, Peligrad and Rio [14]). Efforts in the non-logarithmic scale for dependent variables were made by Grama [7], Grama and Haeusler [8] for martingales, Wu and Zhao [32] for stationary processes, Tang [30] for negatively dependent variables or Liu [12] for negatively dependent heavy tailed variables.

In this paper we present a moderate deviation in the non-logarithmic scale for sums of associated random variables. In Section 2 we give some definitions and recall some auxiliary results, in Section 3 we prove a first moderate deviation based on an assumption depending on a Gaussian approximation. In Section 4 we discuss this approximation issue, giving a general moderate deviation based on more natural assumptions. Finally, in Section 5 we give an application to moving averages of our results.

\section{Framework and auxiliary results}

To define appropriately our framework let $X_{n}, n \geq 1$, be strictly stationary, centered and associated random variables with finite variances. Denote $S_{n}=X_{1}+\cdots+X_{n}$ and 
$s_{n}^{2}=\mathrm{E} S_{n}^{2}$. Recall that association means that for any $m \geq 1$ and any two real-valued coordinatewise nondecreasing functions $f$ and $g$,

$$
\operatorname{Cov}\left(f\left(X_{1}, \ldots, X_{m}\right), g\left(X_{1}, \ldots, X_{m}\right)\right) \geq 0,
$$

whenever this covariance exists. For basic results on associated random variables, please refer to the monographs Bulinski, Shashkin [4], Oliveira [22] or Prakasa Rao [26]. It is well known that the covariance structure of associated random variables characterizes their asymptotics, so it is natural to seek assumptions on the covariances.

A common assumption when proving Central Limit Theorems is $\frac{1}{n} s_{n}^{2} \longrightarrow \sigma^{2}>0$ (see, for example, Newman and Wright [19, 20] or Oliveira and Suquet [23, 24]), so we will be assuming that this is fulfilled in the sequel. Notice this assumption implies that $s_{n}^{2} \sim n \sigma^{2}$. Finally, define the Cox-Grimmett coefficients, commonly used to control dependence for associated variables:

$$
u(n)=\sum_{k=n}^{\infty} \operatorname{Cov}\left(X_{1}, X_{k}\right) .
$$

Our proof will rely on a suitable approximation to independent variables that will be chosen so they satisfy the moderate deviation we want to extend. We quote next a result by Frolov [6], providing a moderate deviation for triangular arrays of row-wise independent random variables. This will be the tool to prove the moderate deviation for the approximating variables.

Theorem 2.1 (Theorem 1.1 in Frolov [6]). Let $X_{n, k}, k=1, \ldots, k_{n}, n \geq 1$, be an array of row-wise independent variables with $F_{n, k}(y)=\mathrm{P}\left(X_{n, k} \leq y\right), \mathrm{E} X_{n, k}=0$ and $\mathrm{E} X_{n, k}^{2}=\sigma_{n, k}^{2}<\infty$. Denote $T_{n}=\sum_{k=1}^{k_{n}} X_{n, k}$ and $B_{n}=\sum_{k=1}^{k_{n}} \sigma_{n, k}^{2}$. For $q>2$, let $\beta_{n, k}=\int_{0}^{\infty} y^{q} F_{n, k}(d y)<+\infty$, and define

$$
M_{n}=\sum_{k=1}^{k_{n}} \beta_{n, k} \quad \text { and } \quad L_{n}=B_{n}^{-q / 2} M_{n} .
$$

Assume that $L_{n} \longrightarrow 0$, and that, for each $\varepsilon>0$,

$$
\Lambda_{n}(x)=x^{4} B_{n}^{-1} \sum_{k=1}^{n} \int_{-\infty}^{-\varepsilon \sqrt{B_{n}} / x^{5}} y^{2} F_{n, k}(d y) \longrightarrow 0 .
$$

If $x \longrightarrow+\infty$ such that $x^{2}-2 \log \left(L_{n}^{-1}\right)-(q-1) \log \log \left(L_{n}^{-1}\right) \longrightarrow-\infty$ then

$$
\mathrm{P}\left(T_{n} \geq x B_{n}^{1 / 2}\right) \sim \frac{1}{\sqrt{2 \pi} x} e^{-x^{2} / 2} .
$$


Remark that, using standard Gaussian approximations, from the conclusion of this theorem follows easily that $\mathrm{P}\left(T_{n} \geq x B_{n}^{1 / 2}\right)=(1-\Phi(x))(1+o(1))$, where $\Phi$ stands for the distribution function of a standard Gaussian variable.

Finally, we will be dealing with integration of squares of sums of random variables that we will need to decompose. The following result describes how we can do this and control the original integral.

Lemma 2.2 (Lemma 4 in Utev [31]). Let $U_{n}, n \geq 1$, be random variables. Then, for every $\varepsilon>0$ and $n \geq 1$,

$$
\int_{\left\{\left|\sum_{i=1}^{n} U_{i}\right|>\varepsilon n\right\}}\left(\sum_{i=1}^{n} U_{i}\right)^{2} d P \leq 2 n \sum_{i=1}^{n} \int_{\left\{\left|U_{i}\right|>\varepsilon / 2\right\}} U_{i}^{2} d P .
$$

\section{A general moderate deviation}

We now state a moderate deviation for associated random variables. Besides moment conditions we will require a suitable decrease rate on the Cox-Grimmett coefficients (2). To state and prove our first result we need some preparatory definitions. Consider an increasing sequence of integers $p_{n}<\frac{n}{2}$ and define $r_{n}$ as the largest integer that is less or equal to $\frac{n}{2 p_{n}}$. Decompose $S_{n}=X_{1}+\cdots+X_{n}$ into blocks, each summing $p_{n}$ variables. For this purpose, define

$$
Y_{j, n}=\sum_{\ell=(j-1) p_{n}+1}^{j p_{n}} X_{\ell}, \quad j=1, \ldots, 2 r_{n},
$$

which obviously verify

$$
S_{n}=Y_{1, n}+\cdots+Y_{2 r_{n}, n}+\sum_{\ell=2 r_{n} p_{n}+1}^{n} X_{\ell} .
$$

The final term is a residual block summing at most $2 p_{n}-1$ variables. Finally, put

$$
Z_{n, o d}=\sum_{j=1}^{r_{n}} Y_{2 j-1, n} \quad \text { and } \quad Z_{n, e v}=\sum_{j=1}^{r_{n}} Y_{2 j, n} .
$$

Define now a family of coupling variables: $Y_{j, n}^{*}, j=1, \ldots, r_{n}$, are independent random variables such that $Y_{j, n}^{*}$ has the same distribution as $Y_{j, n}$. Remark that, if the original 
variables $X_{n}$ are strictly stationary, the $Y_{j, n}^{*}, j=1, \ldots, r_{n}$, are identically distributed. Moreover, in such case, $E\left(Y_{j, n}^{*}\right)^{2}=s_{p_{n}}^{2}$. Further, denote

$$
Z_{n, o d}^{*}=\sum_{j=1}^{r_{n}} Y_{2 j-1, n}^{*} \quad \text { and } \quad Z_{n, e v}^{*}=\sum_{j=1}^{r_{n}} Y_{2 j, n}^{*} .
$$

Finally, to complete the notation description, given a set $A$, we will denote by $\mathbb{I}_{A}$ its indicator function.

Theorem 3.1. Let $X_{n}, n \geq 1$, be strictly stationary, centered and associated random variables. Let $S_{n}=X_{1}+\cdots+X_{n}, s_{n}^{2}=\mathrm{E} S_{n}^{2}$. Assume that

(A1) the random variables $X_{n}$ have finite moments of order $q>2$;

(A2) $x_{n} \longrightarrow+\infty$ such that $\lim \sup _{n \rightarrow+\infty} \frac{x_{n}}{\log n}=2 \gamma<q-2$;

(A3) $\frac{1}{n} s_{n}^{2} \longrightarrow \sigma^{2}$ for some $\sigma^{2}<\infty$;

(A4) $u(n)=O\left(n^{-\theta}\right)$, where $\theta>(1+3 \gamma) \max \left(2+\frac{4}{q-2}, 2+\frac{4 \gamma+2}{q-2 \gamma-2}\right)$;

(A5) $\left|\mathrm{P}\left(S_{n}>x_{n} s_{n}\right)-\mathrm{P}\left(Z_{n, o d}>x_{n} \frac{s_{n}}{\sqrt{2}}\right)\right|=O\left(n^{-\gamma}(\log n)^{-1 / 2}\right)$.

Then

$$
\mathrm{P}\left(S_{n}>x_{n} s_{n}\right)=\left(1-\Phi\left(x_{n}\right)\right)(1+o(1)) .
$$

Proof. The proof of the theorem follows the more or less classical steps after the decomposition of $S_{n}$ into blocks and coupling these blocks with variables with the same distribution but independent: 1 . prove the moderate deviation for the coupling variables; 2. control the difference between the original blocks and the coupling ones; 3 . prove the residual block converges to zero at the appropriate rate; 4 . finally, approximate the convenient tail probabilities. To complete this plan we need to be more specific about the sequence $p_{n}$ used for the construction of the blocks, assuming that $p_{n} \sim n^{1-\alpha}$, where $\frac{1}{1-\alpha}>\max \left(2+\frac{4}{q-2}, 2+\frac{4 \gamma+2}{q-2 \gamma-2}\right)$ or, equivalently,

$$
\max \left(\frac{1}{2}+\frac{1}{q}, \frac{1}{2}+\frac{1+2 \gamma}{2(q-1)}\right)<\alpha<1
$$

(remark that the assumption on $\gamma$ in (A2) ensures that this interval is nonempty and remember that $\left.p_{n}<\frac{n}{2}\right)$. Finally, to complete the preliminary preparations, ater picking $\alpha$ in the given interval, choose $\gamma^{\prime}>\gamma$ such that $\theta=\frac{1+3 \gamma^{\prime}}{1-\alpha}$ fulfilling (A4). 
Step 1. To accomplish this step we apply Theorem 2.1 to the random variables $Y_{j, n}^{*}$ defining each of the summations $Z_{n, o d}^{*}$ and $Z_{n, e v}^{*}$. We shall concentrate on $Z_{n, o d}^{*}$, as the other summation is analogous. Now, as mentioned above, $Z_{n, o d}^{*}$ is a sum of identically distributed random variables. It follows from (A1), that the moment assumption required by Theorem 2.1 on the variables $Y_{j, n}^{*}$ is satisfied. Referring to the notation of Theorem 2.1, we have $B_{n}=r_{n} s_{p_{n}}^{2} \sim n \sigma^{2} / 2$ (this corresponds to our $\left.s_{n}^{2}\right), M_{n}=r_{n} \mathrm{E}\left(Y_{j, n}^{q} \mathbb{I}_{Y_{j, n} \geq 0}\right)$ and $L_{n}=r_{n} B_{n}^{-q / 2} \mathrm{E}\left(Y_{j, n}^{q} \mathbb{I}_{Y_{j, n} \geq 0}\right) \sim r_{n} n^{-q / 2} p_{n}^{q}=n^{(1-\alpha)(q-1)+1-q / 2}$. The exponent in this last expression is rewritten as $\frac{q}{2}-\alpha(q-1)<-\gamma<0$, as follows from (5), thus $L_{n} \longrightarrow 0$, as required by Theorem 2.1. Moreover, $x_{n}^{2}-2 \log L_{n}^{-1} \sim x_{n}^{2}-\log n^{2 \alpha(q-1)-q}$. Again from (5), we have that $\alpha>\frac{1}{2}+\frac{1+2 \gamma}{2(q-1)}$, so it follows that $2 \alpha(q-1)-q>2 \gamma$, thus $x_{n}^{2}-2 \log L_{n}^{-1} \longrightarrow-\infty$, hence satisfying the assumption on $x_{n}$ in Theorem 2.1.

Concerning (3), a Lindeberg like assumption in Theorem 2.1, notice that when applied to the $Y_{j, n}^{*}$ variables, remembering that $B_{n} \sim n \sigma^{2} / 2$ and that all the terms in the summation are identical, it may be rewritten as

$$
x_{n}^{4} E\left(Y_{j, n}^{2} \mathbb{I}_{\left(-\infty,-\varepsilon s_{n} / x_{n}^{5}\right)}\left(Y_{j, n}\right)\right) \longrightarrow 0 .
$$

(We do not include the $*$ as the mathematical expectation above only depends on the moments of each variable). Of course, we may replace $s_{n}$ by $n^{1 / 2} \sigma$. Enlarging the integration set, we obviously have the upper bound

$$
x_{n}^{4} E\left(Y_{j, n}^{2} \mathbb{I}_{\left(-\infty,-\varepsilon n^{1 / 2} \sigma / x_{n}^{5}\right)}\left(Y_{j, n}\right)\right) \leq x_{n}^{4} E\left(Y_{j, n}^{2} \mathbb{I}_{\left|Y_{j, n}\right|>\varepsilon n^{1 / 2} \sigma / x_{n}^{5}}\right) .
$$

The integrand above is the square of a sum of random variables, so we need to separate the random variables in this square. This may be accomplished using Lemma 2.2. Remembering that the $X_{n}$ variables are identically distributed, one easily obtains that

$$
\begin{aligned}
x_{n}^{4} E & \left(Y_{j, n}^{2} \mathbb{I}_{\left|Y_{j, n}\right|>\varepsilon n^{1 / 2} \sigma / x_{n}^{5}}\right) \\
& \leq 2 x_{n}^{4} p_{n}^{2} \int_{\left\{\left|X_{i}\right|>\varepsilon n^{1 / 2} \sigma /\left(2 p_{n} x_{n}^{5}\right)\right\}} X_{i}^{2} d P \\
& \leq 2 x_{n}^{4} p_{n}^{2}\left(E\left|X_{1}\right|^{q}\right)^{2 / q}\left(P\left(\left|X_{1}\right|>\frac{\varepsilon n^{1 / 2} \sigma}{2 p_{n} x_{n}^{5}}\right)\right)^{1-2 / q} \\
& \leq 2 x_{n}^{4} p_{n}^{2}\left(E\left|X_{1}\right|^{q}\right)^{2 / q}\left(E\left|X_{1}\right|^{q}\left(\frac{2 p_{n} x_{n}^{5}}{\varepsilon n^{1 / 2} \sigma}\right)^{q}\right)^{1-2 / q} \\
& =2 E\left|X_{1}\right|^{q} \frac{2^{q-2} p_{n}^{q} x_{n}^{5 q-6}}{\varepsilon^{q-2} n^{(q-2) / 2} \sigma^{q-2}} .
\end{aligned}
$$


Taking into account (A2), $x_{n}^{2}$ grows to infinity at most at a logarithmic rate, thus the asymptotic behaviour of the term above is driven by the polynomial factors. We have chosen $p_{n} \sim n^{1-\alpha}$, so it follows that

$$
\frac{p_{n}^{q} x_{n}^{5 q-6}}{n^{(q-2) / 2}} \lesssim \frac{p_{n}^{q}(\log n)^{5 q / 2-3}}{n^{(q-2) / 2}} \sim n^{q(1 / 2-\alpha)+1}(\log n)^{5 q / 2-3} \longrightarrow 0,
$$

since $q\left(\frac{1}{2}-\alpha\right)+1<0$, taking into account (5). Then, from Theorem 2.1 it follows that $\left(\right.$ remember $\left.\mathrm{E}\left(Y_{j, n}^{2}\right)=s_{p_{n}}^{2}\right)$

$$
P\left(Z_{n, o d}^{*}>x_{n} s_{p_{n}} \sqrt{r_{n}}\right) \sim \frac{1}{\sqrt{2 \pi} x_{n}} e^{-x_{n}^{2} / 2} .
$$

Step 2. Denote by $G_{1}$ the distribution function of $Z_{n, \text { od }}$, by $G_{2}$ the distribution function when the summands are assumed independent, that is, the distribution function of $Z_{n, o d}^{*}$, and by $\varphi_{1}$ and $\varphi_{2}$ the corresponding characteristic functions:

$$
\varphi_{1}(t)=E\left(e^{i t Z_{n, o d}}\right) \quad \text { and } \quad \varphi_{2}(t)=\prod_{j=1}^{r_{n}} E\left(e^{i t Y_{2 j-1, n}}\right) .
$$

The classical Berry-Esséen inequality states that

$$
\sup _{x \in \mathbb{R}}\left|G_{1}(x)-G_{2}(x)\right| \leq c_{1} \int_{-T}^{T} \frac{\left|\varphi_{1}(t)-\varphi_{2}(t)\right|}{|t|} d t+\frac{c_{2}}{T}, \quad \text { for every } T>0,
$$

where $c_{1}$ and $c_{2}$ are constants independent of $T$. It follows from Newman's inequality for characteristic functions of associated variables (Theorem 1 in Newman [18]) that

$$
\left|\varphi_{1}(t)-\varphi_{2}(t)\right| \leq \frac{t^{2}}{2} \sum_{j \neq k} \operatorname{Cov}\left(Y_{2 j-1, n}, Y_{2 k-1, n}\right) .
$$

As the $X_{n}$ are stationary, it still follows that

$$
\sum_{j \neq k} \operatorname{Cov}\left(Y_{2 j-1, n}, Y_{2 k-1, n}\right) \leq n \sum_{\ell=p_{n}+2}^{+\infty} \operatorname{Cov}\left(X_{1}, X_{\ell}\right)=n u\left(p_{n}+2\right) \leq n u\left(p_{n}\right),
$$

referring to the Cox-Grimmett coefficients, as the covariances are nonnegative. Inserting this into the Berry-Esséen bound one finds

$$
\sup _{x \in \mathbb{R}}\left|G_{1}(x)-G_{2}(x)\right| \leq \frac{c_{1}}{2} \int_{-T}^{T} n u\left(p_{n}\right)|t| d t+\frac{c_{2}}{T} \leq \frac{c_{1}}{2} n u\left(p_{n}\right) T^{2}+\frac{c_{2}}{T},
$$

So, choosing $T \sim\left(n u\left(p_{n}\right)\right)^{-1 / 3}$, we find an upper bound of order $\left(n u\left(p_{n}\right)\right)^{1 / 3}$. Using now the choice $p_{n} \sim n^{1-\alpha}$ and taking into account (5), it follows that $\left(n u\left(p_{n}\right)\right)^{1 / 3} \sim n^{-\gamma^{\prime}}$. 
Given the behaviour of $x_{n}$ described in assumption (A2), it follows that $x_{n}^{-1} e^{-x_{n}^{2} / 2} \sim$ $n^{-\gamma}(\log n)^{-1 / 2}$, hence, as $\gamma^{\prime}>\gamma$, we have $\left(n u\left(p_{n}\right)\right)^{1 / 3}=O\left(x_{n}^{-1} e^{-x_{n}^{2} / 2}\right)$, which controls the convergence rate of the approximation between the actual variables and the coupling ones.

Step 3. We prove that the residual block defines probabilities that converge to zero faster than the terms considered in the previous steps. Remember that it follows from (A3) that $s_{n} \sim n^{1 / 2} \sigma^{2}$. Thus, as the variables $X_{\ell}$ are identically distributed,

$$
\begin{aligned}
& \mathrm{P}\left(\sum_{\ell=2 r_{n} p_{n}+1}^{n} X_{\ell}>x_{n} s_{n}\right) \\
& \quad \leq \sum_{\ell=2 r_{n} p_{n}}^{n} \mathrm{P}\left(X_{\ell}>\frac{x_{n} n^{1 / 2} \sigma}{n-2 r_{n} p_{n}}\right) \leq \frac{\left(n-2 r_{n} p_{n}\right)^{q+1}}{x_{n} n^{q / 2} \sigma^{q}} \mathrm{E}\left|X_{1}\right|^{q} .
\end{aligned}
$$

As $x_{n}^{2}$ grows at most at a logarithmic, it is enough to verify that

$$
\frac{\left(n-2 r_{n} p_{n}\right)^{q+1}}{n^{q / 2}} \leq \frac{2^{q+1} p_{n}^{q+1}}{n^{q / 2}} \sim n^{(q+1)(1-\alpha)-q / 2} .
$$

Now $(q+1)(1-\alpha)-\frac{q}{2}>(q-1)(1-\alpha)-\frac{q}{2}>\gamma$, as follows from (5), so there exists $\gamma^{*} \in\left(\gamma,(q+1)(1-\alpha)-\frac{q}{2}\right)$ such that

$$
\mathrm{P}\left(\sum_{\ell=2 r_{n} p_{n}+1}^{n} X_{\ell}>x_{n} s_{n}\right)=O\left(n^{-\gamma^{*}}\right)=O\left(x_{n}^{-1} e^{-x_{n}^{2} / 2}\right) .
$$

Step 4. In the previous steps we controlled the behaviour of $\mathrm{P}\left(Z_{n, \text { od }}>x_{n} s_{p_{n}} \sqrt{r_{n}}\right)$, but we are interested in probabilities of the form $\mathrm{P}\left(S_{n}>x_{n} s_{n}\right)$. Noting that $s_{p_{n}} \sqrt{r_{n}} \sim \frac{s_{n}}{\sqrt{2}}$, the difference between these two terms is controlled at the appropriate convergence rate by (A5).

Remark 3.2. It is obvious from the proof above that assumption (A4) can be relaxed considering logarithmic factors instead of the purely polynomial order $\theta$. Indeed, the result in Theorem 3.1 still holds if we replace this assumption by

(A4.1) $u(n)=O\left(n^{-\theta}(\log n)^{-3 / 2}\right)$, where $\theta>(1+3 \gamma) \max \left(2+\frac{4}{q-2}, 2+\frac{4 \gamma+2}{q-2 \gamma-2}\right)$.

Remark 3.3. We give an example showing that (A5) is indeed achievable. We have assumed the $X_{n}$ to be stationary, so $Z_{n, o d}$ and $Z_{n, e v}$ have the same distribution. Assume further, for simplicity, that $S_{n}=Z_{n, o d}+Z_{n, e v}$, that is, the residual term does not exist 
(remember we have already shown that this residual term is negligible), and $\left(Z_{n, o d}, Z_{n, e v}\right)$ is Gaussian centered with $\operatorname{Var}\left(Z_{n, o d}\right)=\operatorname{Var}\left(Z_{n, e v}\right)=a_{n}$ and $\operatorname{Cov}\left(Z_{n, o d}, Z_{n, e v}\right)=\rho_{n}$. Remark that, due to the association, we have $\rho_{n} \geq 0$. Then, $S_{n}$ is Gaussian with mean 0 and variance $s_{n}^{2}=2 a_{n}+2 \rho_{n}$. So, denoting by $Z$ a standard Gaussian random variable, we have that

$$
\begin{aligned}
& \left|\mathrm{P}\left(S_{n}>x s_{n}\right)-\mathrm{P}\left(Z_{n, o d}>x \frac{s_{n}}{\sqrt{2}}\right)\right| \\
& \quad=\left|\mathrm{P}(Z>x)-\mathrm{P}\left(Z>\left(1+\frac{\rho_{n}}{a_{n}}\right)^{1 / 2} x\right)\right| \leq \mathrm{P}(Z>x) .
\end{aligned}
$$

As we have already remarked, when $x \longrightarrow+\infty$,

$$
\mathrm{P}(Z>x) \sim \frac{1}{\sqrt{2 \pi} x} e^{-x^{2} / 2} .
$$

Using this approximation on the previous it follows that, for some constant $C_{1}>0$,

$$
\left|\mathrm{P}\left(S_{n}>x s_{n}\right)-\mathrm{P}\left(Z_{n, o d}>x s_{n}\right)\right| \leq C_{1} x^{-1} e^{-x^{2} / 2} .
$$

If we now take $x$ satisfying (A2), this means that (A5) is fulfilled.

Remark 3.4. Note that the assumption on the Cox-Grimmett coefficients, (A4), is much milder than what was assumed in Henriques and Oliveira [9] to prove a large deviation principle: $\operatorname{Cov}\left(X_{1}, X_{n}\right)=a_{0} \exp \left(-n(\log n)^{1+a}\right)$, where $a_{0}>0$ and $a>0$.

Let us get back to the discussion about assumption (A5), seeking for more a natural sufficient condition. According to Remark 3.3, when the distributions are Gaussians, (A5) is satisfied. So, one way to look for more natural conditions is to try to control the distance with respect to Gaussian distributions using Berry-Esséen bounds.

Theorem 3.5. Let $X_{n}, n \geq 1$, be strictly stationary, centered and associated random variables. Let $S_{n}=X_{1}+\cdots+X_{n}, s_{n}^{2}=\mathrm{E} S_{n}^{2}$. Assume that (A1)-(A4) in Theorem 3.1 are satisfied with $q \geq 3$ and $\gamma<\frac{1}{5}$. Then (4) holds.

Proof. We need to verify that (A5) is satisfied. For this purpose introduce Gaussian centered variables $\widehat{S}_{n}, \widehat{Z}_{n, o d}$ and $\widehat{Z}_{n, e v}$ with variances $\mathrm{E} S_{n}^{2}$, E $Z_{n, o d}^{2}$ and $\mathrm{E} Z_{n, e v}^{2}$, respec- 
tively, and such that $\operatorname{Cov}\left(\widehat{Z}_{n, o d}, \widehat{Z}_{n, e v}\right)=\operatorname{Cov}\left(Z_{n, o d}, Z_{n, e v}\right)$, and decompose

$$
\begin{aligned}
\left|\mathrm{P}\left(S_{n}>x_{n} s_{n}\right)-\mathrm{P}\left(Z_{n, \text { od }}>x_{n} \frac{s_{n}}{\sqrt{2}}\right)\right| \\
\leq\left|\mathrm{P}\left(S_{n}>x_{n} s_{n}\right)-\mathrm{P}\left(\widehat{S}_{n}>x_{n} s_{n}\right)\right| \\
\quad+\left|\mathrm{P}\left(\widehat{S}_{n}>x_{n} s_{n}\right)-\mathrm{P}\left(\widehat{Z}_{n, o d}>x_{n} \frac{s_{n}}{\sqrt{2}}\right)\right| \\
\quad+\left|\mathrm{P}\left(\widehat{Z}_{n, \text { od }}>x_{n} \frac{s_{n}}{\sqrt{2}}\right)-\mathrm{P}\left(Z_{n, \text { od }}>x_{n} \frac{s_{n}}{\sqrt{2}}\right)\right| .
\end{aligned}
$$

Remark 3.3 shows that the middle term above is of order $n^{-\gamma}(\log n)^{-1 / 2}$. As the variables satisfy the Central Limit Theorem, the remaining terms may be bounded by the BerryEsséen inequality. Now, taking into account Corollary 4.24 in Oliveira [22], the convergence rate for these terms is of order $n^{-1 / 5}$. Hence, $\left|\mathrm{P}\left(S_{n}>x_{n} s_{n}\right)-\mathrm{P}\left(Z_{n, o d}>x_{n} \frac{s_{n}}{\sqrt{2}}\right)\right|$ is of the same order as the slowest term, that is $n^{-\gamma}(\log n)^{-1 / 2}$, thus (A5) is satisfied, so the conclusion of Theorem 3.1 holds, that is, (4) is verified.

\section{Main result}

The result stated in Theorem 3.5 is a sort of a worst case scenario concerning the approximation to the Gaussian distribution. We may improve on our Theorem 3.1 if we are more precise about the convergence rate in assumption (A3). To accomplish this we need first to prove an adapted version of the Berry-Esséen bound for the approximation of distribution functions in the Central Limit Theorem.

Theorem 4.1. Let $X_{n}, n \geq 1$, be strictly stationary, centered and associated random variables with finite moments of order 3. Let $S_{n}=X_{1}+\cdots+X_{n}, s_{n}^{2}=\mathrm{E} S_{n}^{2}$ and assume that $\frac{1}{n} s_{n}^{2} \longrightarrow \sigma^{2}<\infty$. If $p_{n}$ and $r_{n}$ are sequences as defined in the beginning of Section 3, then, for n large enough,

$$
\begin{aligned}
& \sup _{x \in \mathbb{R}}\left|\mathrm{P}\left(S_{n} \leq x s_{n}\right)-\Phi(x)\right| \\
& \quad \leq T^{2}\left(1-\frac{2 r_{n} s_{p_{n}}^{2}}{s_{n}^{2}}\right)+\frac{24}{\pi \sqrt{2 \pi} T}+4 \sqrt{\pi} c_{1}^{\prime} e^{c_{1}^{\prime} /\left(2 c_{1}^{2}\right)} \frac{r_{n} \mathrm{E}\left|Y_{1, n}\right|^{3}}{s_{n}^{3}},
\end{aligned}
$$

where $\Phi(\cdot)$ is the distribution function of the standard Gaussian distribution, $T=\frac{s_{p_{n}}^{2} s_{n}}{4 \mathrm{E}\left|Y_{1, n}^{3}\right|}$ and $c_{1}, c_{1}^{\prime}>0$ are constants independent from $n$ and from the distributions of the random variables. 
Proof. Using the classical Berry-Esséen bound we have for every $T>0$ (see, for example, Theorem A.1 in [22]),

$$
\sup _{x \in \mathbb{R}}\left|\mathrm{P}\left(S_{n} \leq x s_{n}\right)-\Phi(x)\right| \leq \frac{1}{\pi} \int_{-T}^{T} \frac{1}{|t|}\left|\varphi_{S_{n}}\left(\frac{t}{s_{n}}\right)-e^{-t^{2} / 2}\right| d t+\frac{24}{\pi \sqrt{2 \pi} T},
$$

where $\varphi_{S_{n}}$ represents that characteristic function of $S_{n}$. To bound the integral above remember that $S_{n}=Y_{1, n}+\cdots+Y_{2 r_{n}, n}$ and add and subtract the terms $\prod_{j=1}^{2 r_{n}} \mathrm{E}^{\frac{i t}{s_{n}} Y_{j, n}}$ and $e^{-r_{n} t^{2} s_{p_{n}}^{2} / s_{n}^{2}}$ inside the absolute value and separate the corresponding three integrals, and that, due to the strict stationarity, the blocks $Y_{j, n}$ have the same distribution as $S_{p_{n}}$. Now, using Newman's inequality for characteristic functions (Theorem 1 in Newman [18]), for the first integral obtained it follows immediately that,

$$
\begin{aligned}
\int_{-T}^{T} & \frac{1}{|t|}\left|\operatorname{E} \exp \left(\frac{i t}{s_{n}} \sum_{j=1}^{2 r_{n}} Y_{j, n}\right)-\prod_{j=1}^{2 r_{n}} \mathrm{E} e^{\frac{i t}{s_{n}} Y_{j, n}}\right| d t \\
& \leq \frac{1}{2} \int_{-T}^{T} \frac{1}{|t|} \sum_{j \neq j^{\prime}} \frac{t^{2}}{s_{n}^{2}} \operatorname{Cov}\left(Y_{j, n}, Y_{j^{\prime}, n}\right) d t=\frac{T^{2}}{2}\left(1-\frac{2 r_{n} s_{p_{n}}^{2}}{s_{n}^{2}}\right) .
\end{aligned}
$$

The third integral is also easily bounded. Indeed, using $\left|e^{x}-e^{y}\right| \leq|x-y|$,

$$
\int_{-T}^{T} \frac{1}{|t|}\left|e^{-r_{n} t^{2} s_{p_{n}}^{2} / s_{n}^{2}}-e^{-t^{2} / 2}\right| d t \leq \frac{T^{2}}{2}\left(1-\frac{2 r_{n} s_{p_{n}}^{2}}{s_{n}^{2}}\right) .
$$

We have thus obtained the first two terms in the upper bound in (6). The remaining integral to analyse is

$$
\int_{-T}^{T} \frac{1}{|t|}\left|\prod_{j=1}^{2 r_{n}} \mathrm{E} e^{\frac{i t}{s_{n}} Y_{j, n}}-e^{-r_{n} t^{2} s_{p_{n}}^{2} / s_{n}^{2}}\right| d t=\int_{-T}^{T} \frac{1}{|t|}\left|\prod_{j=1}^{2 r_{n}} \varphi_{Y_{j, n}}\left(\frac{t}{s_{n}}\right)-e^{-r_{n} t^{2} s_{p_{n}}^{2} / s_{n}^{2}}\right| d t,
$$

where $\varphi_{Y_{j, n}}$ is the characteristic function of $Y_{j, n}$. Let $W_{j}, j=1, \ldots, 2 r_{n}$, be random variables with the same distribution as $Y_{j, n}$ such that these two variables are independent. Then, for each $j=1, \ldots, r_{n}, \mathrm{E}\left(W_{j}-Y_{j, n}\right)=0, \operatorname{Var}\left(W_{j}-Y_{j, n}\right)=2 s_{p_{n}}^{2}$ and $\mathrm{E}\left|W_{j}-y_{j, n}\right|^{3} \leq 8 \mathrm{E}\left|Y_{j, n}\right|^{3}$. Hence, for some $\theta \in(-1,1)$,

$$
\begin{aligned}
\left|\varphi_{Y_{j, n}}\left(\frac{t}{s_{n}}\right)\right|^{2} \varphi_{W_{j}-Y_{j, n}}\left(\frac{t}{s_{n}}\right) & \leq 1-\frac{s_{p_{n}}^{2} t^{2}}{s_{n}^{2}}+\frac{4 \theta}{3} \frac{|t|^{3} \mathrm{E}\left|Y_{j, n}\right|^{3}}{s_{n}^{3}} \\
& \leq \exp \left(-\frac{s_{p_{n}}^{2} t^{2}}{s_{n}^{2}}+\frac{4 \theta}{3} \frac{|t|^{3} \mathrm{E}\left|Y_{j, n}\right|^{3}}{s_{n}^{3}}\right)
\end{aligned}
$$


and

$$
\left|\prod_{j=1}^{2 r_{n}} \varphi_{Y_{j, n}}\left(\frac{t}{s_{n}}\right)\right|^{2} \leq \exp \left(-\frac{2 r_{n} t^{2} s_{p_{n}}^{2}}{s_{n}^{2}}+\frac{8 \theta}{3} \frac{|t|^{3} r_{n} \mathrm{E}\left|Y_{1, n}\right|^{3}}{s_{n}^{3}}\right) .
$$

Assume that $|t| \leq T=\frac{s_{p_{n}}^{2} s_{n}^{2}}{4 \mathrm{E}\left|Y_{1, n}^{3}\right|}$. Then $\frac{8 \theta}{3} \frac{|t|^{3} \mathrm{E}\left|Y_{1, n}\right|^{3}}{s_{n}^{3}} \leq \frac{2 r_{n} t^{2} s_{p_{n}}^{2}}{3 s_{n}^{2}}$, thus

$$
\begin{aligned}
\left|\varphi_{Y_{j, n}}\left(\frac{t}{s_{n}}\right)-e^{-r_{n} t^{2} s_{p_{n}}^{2} / s_{n}^{2}}\right| & \leq \exp \left(-\frac{2 r_{n} t^{2} s_{p_{n}}^{2}}{3 s_{n}^{2}}\right)+\exp \left(-\frac{r_{n} t^{2} s_{p_{n}}^{2}}{s_{n}^{2}}\right) \\
& \leq 2 \exp \left(-\frac{2 r_{n} t^{2} s_{p_{n}}^{2}}{3 s_{n}^{2}}\right) .
\end{aligned}
$$

Another Taylor expansion gives, for some $\theta \in(-1,1)$,

$$
\varphi_{Y_{j, n}}\left(\frac{t}{s_{n}}\right)=1-\frac{t^{2} s_{p_{n}}^{2}}{2 s_{n}^{2}}+\theta \frac{|t|^{3} \mathrm{E}\left|Y_{j, n}\right|^{3}}{6 s_{n}^{3}} .
$$

If we assume now that $|t| \leq \frac{s_{n}}{c_{1}\left(2 r_{n} E\left|Y_{1, n}\right|^{3}\right)^{1 / 3}}$, it follows from the previous inequality that

$$
\left|\varphi_{Y_{j, n}}\left(\frac{t}{s_{n}}\right)-1\right| \leq \frac{1}{2\left(2 r_{n}\right)^{2 / 3} c_{1}^{2}}+\frac{1}{12 c_{1}^{3} r_{n}},
$$

which is, for $n$ large enough, arbitrarily small, thus the characteristic function is bounded away from 0 for $|t| \leq \frac{s_{n}}{c_{1}\left(2 r_{n} E\left|Y_{1, n}\right|^{3}\right)^{1 / 3}}$. Moreover, from (8) and taking into account the upper bound for $|t|$, it follows that

$$
\left|\varphi_{Y_{j, n}}\left(\frac{t}{s_{n}}\right)-1\right|^{2} \leq \frac{t^{4} s_{p_{n}}^{4}}{2 s_{n}^{4}}+\frac{t^{6}\left(\mathrm{E}\left|Y_{j, n}\right|^{3}\right)^{2}}{18 s_{n}^{6}} \leq \frac{|t|^{3} \mathrm{E}\left|Y_{j, n}\right|^{3}}{s_{n}^{3}} \frac{1+18 c_{1}^{2}}{36 c_{1}^{3}} .
$$

As the characteristic functions are bounded away from 0 , we may take their logarithms, for which we find that, for some $\theta, \gamma \in(-1,1)$,

$$
\begin{aligned}
\log \varphi_{Y_{j, n}}\left(\frac{t}{s_{n}}\right) & =-\frac{t^{2} s_{p_{n}}^{2}}{2 s_{n}^{2}}+\theta \frac{|t|^{3} \mathrm{E}\left|Y_{j, n}\right|^{3}}{6 s_{n}^{3}}+\gamma \frac{1+18 c_{1}^{2}}{36 c_{1}^{3}} \frac{|t|^{3} \mathrm{E}\left|Y_{j, n}\right|^{3}}{s_{n}^{3}} \\
& =-\frac{t^{2} s_{p_{n}}^{2}}{2 s_{n}^{2}}+\eta \frac{|t|^{3} \mathrm{E}\left|Y_{j, n}\right|^{3}}{2 s_{n}^{3}}
\end{aligned}
$$

where $\eta=\frac{\theta}{3}+\gamma \frac{1+18 c_{1}^{2}}{18 c_{1}^{3}}$. If we define $c_{1}^{\prime}=\frac{1}{3}+\frac{1+18 c_{1}^{2}}{18 c_{1}^{3}}$, we have $|\eta| \leq c_{1}^{\prime} \leq 1$, for $c_{1}$ conveniently chosen. Summing these bound for the logarithms, we find that

$$
\log \varphi_{S_{n}}\left(\frac{t}{s_{n}}\right)=-\frac{r_{n} t^{2} s_{p_{n}}^{2}}{s_{n}^{2}}+\eta \frac{r_{n}|t|^{3} \mathrm{E}\left|Y_{1, n}\right|^{3}}{s_{n}^{3}},
$$


and

$$
\begin{aligned}
\left|\prod_{j=1}^{2 r_{n}} \mathrm{E} e^{\frac{i t}{s_{n}} Y_{j, n}}-e^{-r_{n} t^{2} s_{p_{n}}^{2} / s_{n}^{2}}\right| & \leq e^{-r_{n} t^{2} s_{p_{n}}^{2} / s_{n}^{2}}\left|e^{c_{1}^{\prime} r_{n}|t|^{3} \mathrm{E}\left|Y_{1, n}\right|^{3} / s_{n}^{3}}-1\right| \\
& \leq \frac{c_{1}^{\prime} r_{n}|t|^{3} \mathrm{E}\left|Y_{1, n}\right|^{3}}{s_{n}^{3}} e^{c_{1}^{\prime} r_{n}|t|^{3} \mathrm{E}\left|Y_{1, n}\right|^{3} / s_{n}^{3}} e^{-r_{n} t^{2} s_{p_{n}}^{2} / s_{n}^{2}} .
\end{aligned}
$$

In order to get an unified upper bound with (7) we choose the constant $c_{1}$ such that, for each $|t|>\frac{s_{n}}{c_{1}\left(2 r_{n} E\left|Y_{1, n}\right|^{3}\right)^{1 / 3}}$,

$$
\frac{c_{1}^{\prime} r_{n}|t|^{3} \mathrm{E}\left|Y_{1, n}\right|^{3}}{s_{n}^{3}} \geq \frac{c_{1}^{\prime}}{2 c_{1}^{3}}=\frac{6 c_{1}^{3}+18 c_{1}^{2}+1}{36 c_{1}^{6}} \geq 2,
$$

which is fulfilled if $c_{1}<.7621$. For such a constant we have thus that

$$
\left|\prod_{j=1}^{2 r_{n}} \mathrm{E} e^{\frac{i t}{s_{n}} Y_{j, n}}-e^{-r_{n} t^{2} s_{p_{n}}^{2} / s_{n}^{2}}\right| \leq \frac{c_{1}^{\prime} r_{n}|t|^{3} \mathrm{E}\left|Y_{1, n}\right|^{3}}{s_{n}^{3}} e^{c_{1}^{\prime} r_{n}|t|^{3} \mathrm{E}\left|Y_{1, n}\right|^{3} / s_{n}^{3}} e^{-r_{n} t^{2} s_{p_{n}}^{2} / s_{n}^{2}}
$$

for every $|t| \leq T=\frac{s_{p_{n}}^{2} s_{n}}{4 \mathrm{E}\left|Y_{1, n}^{3}\right|}$. Taking into account this variation for $t$, it still follows that $\frac{c_{1}^{\prime} r_{n}|t|^{3} \mathrm{E}\left|Y_{1, n}\right|^{3}}{s_{n}^{3}} \leq \frac{c_{1}^{\prime}}{2 c_{1}^{2}}$. Furthermore, as $\frac{1}{n} s_{n}^{2} \longrightarrow \sigma^{2}$ it follows $\frac{r_{n} s_{p_{n}}^{2}}{s_{n}} \longrightarrow 1$, hence, for $n$ large enough, we have $\frac{1}{4}<\frac{r_{n} s_{p_{n}}^{2}}{s_{n}}<1$, so that $e^{-r_{n} t^{2} s_{p_{n}}^{2} / s_{n}^{2}} \leq e^{-t^{2} / 4}$. Inserting this bounds in the integral we find the remaining upper bound in (6).

The first term in (6) leaves the impression that the rate of the convergence $\frac{1}{n} s_{n}^{2} \longrightarrow \sigma^{2}$ can play an important role on simplifying assumption (A5) in Theorem 3.1. This is made explicit in the next statement.

Theorem 4.2. Let $X_{n}, n \geq 1$, be strictly stationary, centered and associated random variables. Let $S_{n}=X_{1}+\cdots+X_{n}, s_{n}^{2}=\mathrm{E} S_{n}^{2}$. Assume that

(B1) the random variables $X_{n}$ have finite moments of order $q \geq 3$;

(B2) $x_{n} \longrightarrow+\infty$ such that $\lim \sup _{n \rightarrow+\infty} \frac{x_{n}}{\log n}=2 \gamma<1$;

(B3) there exists $\sigma^{2}<\infty,\left|\frac{1}{n} s_{n}^{2}-\sigma^{2}\right|=O\left(n^{\beta}\right)$, for some $\beta<\frac{-12 \gamma}{1-2 \gamma}$;

(B4) $u(n)=O\left(n^{-\theta}\right)$, where $\theta \in\left(4+\frac{20 \gamma}{1-2 \gamma}, 4-\beta+(4-\beta) \frac{2 \gamma}{1+\gamma}\right)$

Then

$$
\mathrm{P}\left(S_{n}>x_{n} s_{n}\right)=\left(1-\Phi\left(x_{n}\right)\right)(1+o(1)) .
$$


Proof. As before, we need to define the sizes of the blocks that will be used to decompose $S_{n}$, assuming $p_{n} \sim n^{1-\alpha}$, where

$$
\frac{4}{1-2 \gamma}<\frac{1}{1-\alpha}<\frac{4-\beta}{1+\gamma} \Leftrightarrow \frac{3}{4}+\frac{\gamma}{2}<\alpha<1-\frac{1+\gamma}{4-\beta}
$$

(the assumption on $\beta$ in (B3) ensures this last interval is nonempty). As in the proof of Theorem 3.1, after choosing a suitable $\alpha$ we assume that $\theta=\frac{1+3 \gamma^{\prime}}{1-\alpha}$, for some $\gamma^{\prime}>\gamma$. We may now follow the arguments in the proof of Theorem 3.5. For this purpose introduce, as done when proving Theorem 3.5, Gaussian centered variables $\widehat{S}_{n}, \widehat{Z}_{n, o d}$ and $\widehat{Z}_{n, e v}$ with variances $\mathrm{E} S_{n}^{2}, \mathrm{E} Z_{n, o d}^{2}$ and $\mathrm{E} Z_{n, e v}^{2}$, respectively, and such that $\operatorname{Cov}\left(\widehat{Z}_{n, o d}, \widehat{Z}_{n, e v}\right)=$ $\operatorname{Cov}\left(Z_{n, o d}, Z_{n, e v}\right)$, and decompose

$$
\begin{aligned}
&\left|\mathrm{P}\left(S_{n}>x_{n} s_{n}\right)-\mathrm{P}\left(Z_{n, \text { od }}>x_{n} \frac{s_{n}}{\sqrt{2}}\right)\right| \\
& \leq \mid \mathrm{P}\left(S_{n}>x_{n} s_{n}\right)-\mathrm{P}\left(\widehat{S}_{n}>x_{n} s_{n}\right) \mid \\
&+\left|\mathrm{P}\left(\widehat{S}_{n}>x_{n} s_{n}\right)-\mathrm{P}\left(\widehat{Z}_{n, \text { od }}>x_{n} \frac{s_{n}}{\sqrt{2}}\right)\right| \\
&+\left|\mathrm{P}\left(\widehat{Z}_{n, \text { od }}>x_{n} \frac{s_{n}}{\sqrt{2}}\right)-\mathrm{P}\left(Z_{n, \text { od }}>x_{n} \frac{s_{n}}{\sqrt{2}}\right)\right| .
\end{aligned}
$$

This produces a middle term of order $n^{-\gamma}(\log n)^{-1 / 2}$, as mentioned in Remark 3.3. Now, we have to verify that the approximation to the Gaussian, expressed by the first and third terms above, is, at least, as fast as the rate $n^{-\gamma^{\prime}}$, with $\gamma^{\prime}>\gamma$. Looking at the upper bound in (6), remark that it follows from (B3) that $\left|1-\frac{2 r_{n} s_{p_{n}}^{2}}{s_{n}^{2}}\right|=O\left(n^{\beta(1-\alpha)}\right)$. Moreover, we have $T=\frac{s_{p_{n}}^{2} s_{n}}{4 \mathrm{E}\left|Y_{1, n}\right|^{3}} \sim n^{1 / 2} p_{n}^{-2} \sim n^{2 \alpha-3 / 2} \longrightarrow \infty$, as $\alpha>\frac{3}{4}$. This implies that the two first terms in the upper bound in (6) are of order $T^{2} n^{\beta(1-\alpha)} \sim n^{4 \alpha-3+\beta(1-\alpha)}$ and $T^{-1} \sim n^{3 / 2-2 \alpha}$. It follows from (10) that both $4 \alpha-3+\beta(1-\alpha)<-\gamma^{\prime}$ and $3 / 2-2 \alpha<-\gamma^{\prime}$, thus converging faster than the order $n^{-\gamma}(\log n)^{-1 / 2}$ that comes from the arguments in course of proof of Theorem 3.1. Finally, the last term in the upper bound in (6) is easily verified to be of order $n^{-2 \alpha}$, thus converging faster that $n^{-\gamma^{\prime}}$ as the previous terms, so the proof is concluded.

\section{An application}

As an application of the previous results, consider a moving average model $X_{n}=$ $\sum_{i=1}^{\infty} \phi_{i} \varepsilon_{n-i}$, where the $\varepsilon_{n}$ are independent and identically distributed with mean 0 , variance 1 and finite moments of order $q>2$, and $\phi_{n}>0$, so the $X_{n}, n \geq 1$, are 
associated. Using Hölder inequality it easily follows that $X_{n}$ has finite moment of order $q$, for some $\rho \in(0,1), \sum_{i=1}^{\infty} \phi_{i}^{\rho q}<\infty$ and $\sum_{i=1}^{\infty} \phi_{i}^{(1-\rho) q /(q-1)}<\infty$. Concerning the covariances, whose control is needed in order to verify (A4), it is easily verified that

$$
\operatorname{Cov}\left(X_{1}, X_{n}\right)=\sum_{i=1}^{\infty} \phi_{i} \phi_{n-1+i} \leq\left(\sum_{i=n}^{\infty} \phi_{i}^{\tau}\right)^{1 / \tau}\left(\sum_{i=1}^{\infty} \phi_{i}^{\tau^{\prime}}\right)^{1 / \tau^{\prime}},
$$

where $\tau, \tau^{\prime}>1$ are such that $\tau^{-1}+\left(\tau^{\prime}\right)^{-1}=1$. So, (A4) is verified if the moving average coefficients satisfy, for some $\tau>1$,

$$
\begin{aligned}
& \phi_{n} \longrightarrow 0, \\
& \sum_{i=1}^{\infty} \phi_{i}^{s}<\infty, \text { where } s=\min \left(\rho q, \frac{(1-\rho) q}{q-1}, \frac{\tau}{\tau-1}\right), \rho \in(0,1), \\
& \sum_{i=n}^{\infty} \phi_{i}^{\tau} \sim n^{-\theta \tau}, \theta>(1+3 \gamma) \max \left(2+\frac{4}{q-2}, 2+\frac{4 \gamma+2}{q-2 \gamma-2}\right) .
\end{aligned}
$$

Assume now that the coefficients verify $\phi_{n} \sim n^{-a}$, for some $a>0$. We need to adjust the choice of the decrease rate, that is, the exponent $a$, in order to meet the requirements discussed above. To have the appropriate finite moment of order $q$ for the $X_{n}$ we need to ensure the convergence of the above mentioned series. This follows if we can choose $\rho \in(0,1)$ such that $a \rho q>1$ and $\frac{(1-\rho) a q}{q-1}>1$, that is $\frac{1}{a q}<\rho<1-\frac{a q}{q-1}$. Such a choice is always possible as soon as $a>1$. Inserting now the behaviour of the $\phi_{i}$ in (11) it follows that $\operatorname{Cov}\left(X_{1}, X_{n}\right) \sim n^{-(a+1 / \tau)}$, so that the Cox-Grimmett coefficient $u(n) \sim n^{-(a+1+1 / \tau)}$, where $\tau>1$ is arbitrarily chosen. Thus, in order to verify (A4) we must require $a+1+\frac{1}{\tau}>(1+3 \gamma) \max \left(2+\frac{4}{q-2}, 2+\frac{4 \gamma+2}{q-2 \gamma-2}\right)$, where $0<\gamma<\frac{q}{2}-1$ and $q>2$. So, finally, taking into account the liberty to choose $\tau$, it is enough to require that $a>(1+3 \gamma) \max \left(2+\frac{4}{q-2}, 2+\frac{4 \gamma+2}{q-2 \gamma-2}\right)-2$. A condition based on the more generally usable Theorem 4.2 would require $4-\beta+(4-\beta) \frac{2 \gamma}{1+\gamma}>a+1+\frac{1}{\tau}>4+\frac{20 \gamma}{1-2 \gamma}$ (remember that $\left.\beta<\frac{-12 \gamma}{1-2 \gamma}<0\right)$ or, using the liberty to choose $\tau, 3-\beta+(4-\beta) \frac{2 \gamma}{1+\gamma}>a>2+\frac{20 \gamma}{1-2 \gamma}$ and we should remember that in this case we must have $0<\gamma<\frac{1}{2}$ and $q \geq 3$.

\section{Acknowledgment}

The authors would like to thank the referee for their invaluable comments and suggestions that helped improving on earlier versions of the paper. 
[1] Amosova, N., Limit theorems for the probabilities of moderate deviations (in Russian), Vestnik Leningrad. Univ. No. 13, Mat. Meth. Astronom. Vyp. 3 (1972), 5-14.

[2] Bryc, W., On large deviations for uniformly mixing sequences, Stoch. Proc. and Appl. 41 (1992), 191-202.

[3] Bryc, W., Dembo, A., Large deviations and strong mixing, Ann. Inst. Henri Poincaré 32 (1996), 549-569.

[4] Bulinski, A., Shashkin, A., Limit theorem for associated random fields and relatied systems, World Scientific, Hackensack, NJ, 2007.

[5] Dembo, A., Zeitouni, O., Large Deviations techniques and applications, Springer, New York, 1998.

[6] Frolov, A.N., On probabilities of moderate deviations of sums for independent random variables, J. Math. Sci. 127 (2005), 1787-1796.

[7] Grama, I., On moderate deviations for martingales, Ann. Probab. 25 (1997), 152-183.

[8] Grama, I., Haeusler, E., An asymptotic expansion for probabilities of moderate deviations for multivariate martingales, J. Theoret. Probab. 19 (2006), 1-44.

[9] Henriques, C., Oliveira, P.E., Large deviations for the empirical mean of associated random variables, Statist. Probab. Letters 78 (2008), 594-598.

[10] Ibragimov, I., Linnik, Yu., Independent and stationary sequences of random variables, WoltersNoordhoff Publishing, Groningen, 1971.

[11] Linnik, Yu., Limit Theorems for Sums of Independent Variables Taking into Account Large Deviations I, II, III, Theory Probab. Appl. 6 (1961), 131-148, 345-360; 7 (1962), 115-129.

[12] Lui, L., Precise large deviations for dependent random variables with heavy tails, Statist. Probab. Letters 79 (2009), 1290-1298.

[13] Merlevède, F., Peligrad, M., Rio, E., Bernstein inequality and moderate deviations under strong mixing conditions, High dimensional probability V: the Luminy volume, 273-292, Inst. Math. Stat. Collect., 5, Inst. Math. Statist., Beachwood, OH, 2009.

[14] Merlevède, F., Peligrad, M., Rio, E., A Bernstein type inequality and moderate deviations for weakly dependent sequences, Probab. Theory Related Fields 151 (2011), 435-474.

[15] Nagaev, A., Limit theorems on large deviations under violation of the Cramér condition, Izv. Akad. Mauk Uz.SSR, Ser. fiz-mat. nauk 6 (1969), 17-22.

[16] Nagaev, S., Some Limit Theorems for Large Deviations, Theory Probab. Appl. 10 (1965), $214-235$.

[17] Nagaev, S., Large deviations of sums of independent random variables, Ann. Probab. 7 (1979), $745-689$.

[18] Newman, C.M., Normal fluctuations and the FKG systems, Comm. Math. Phys. 74 (1980), 119-128.

[19] Newman, C.M., Wright, A., An invariance principle for certain dependent sequence, Ann. Probab. 9 (1981), 671-675.

[20] Newman, C.M., Wright, A., Associated random variables and martingale inequalities, Z. Wahrsch. Verw. Gebiete 59 (1982), 361-371.

[21] Nummelin, E., Large deviations for functionals of stationary processes, Probab. Theory Rel Fields 86 (1990), 387-401.

[22] Oliveira, P.E., Asymptotics for Associated Random Variables, Springer, Heidelberg, 2012.

[23] Oliveira, P.E., Suquet, Ch., $L^{2}[0,1]$ weak convergence of the empirical process for dependent variables, Actes des XVèmes Rencontres Franco-Belges de Statisticiens (Ondelettes et Statistique), Eds: Antoniadis, A., Oppenheim, G., Lecture Notes in Statistics 103, 331-344, 1995.

[24] Oliveira, P.E., Suquet, Ch., An $L^{2}[0,1]$ invariance principle for LPQD random variables, Port. Math. 53 (1996), 367-379.

[25] Peligrad, M., Sang, H., Zhong, Y., Wu, W., Exact Moderate and Large Deviations for Linear Processes, Statist. Sinica (2012) doi:10.5705/ss.2012.161.

[26] Prakasa Rao, B.L.S., Associated sequences, demimartingales and nonparametric inference, Birkhäuser/Springer, Basel, 2012.

[27] Rozovski, L., Limit Theorems on Large Deviations in a Narrow Zone, Theory Probab. Appl. 26 (1982), 834-845.

[28] Rubin, H., Sethuraman, J., Probabilites of moderate deviations, Sankhyā Ser. A 27 (1965), 325-346.

[29] Slastnikov, A., Limit Theorems for Moderate Deviation Probabilities, Theory Probab. Appl. 23 (1979), 322-340.

[30] Tang, Q., Insensitivity to negative dependence of the asymptotic behavior of precise large deviations, Electron. J. Probab. 11 (2006), 107-120.

[31] Utev, S.A., On the central limit theorem for $\varphi$-mixing arrays of random variables, Th. Probab. Appl. 35 (1990), 131-139.

[32] Wu, W., Zhao, Z., Moderate deviations for stationary processes, Statist. Sinica 18 (2008), 769-782. 